\title{
Curvelet analysis of asteroseismic data
}

\section{Method description and application to simulated sun-like stars}

\author{
P. Lambert ${ }^{1,2}$, S. Pires ${ }^{3,1}$, J. Ballot ${ }^{2,4}$, R. A. García ${ }^{1,2}$, J.-L. Starck ${ }^{3,1}$, and S. Turck-Chièze ${ }^{1,2}$ \\ 1 DSM/DAPNIA/Service d'Astrophysique, CEA/Saclay, 91191 Gif-sur-Yvette Cedex, France \\ e-mail: lambertp@cea.fr \\ 2 AIM - Unité Mixte de Recherche CEA, CNRS, Université Paris VII - UMR 7158, CEA/Saclay, 91191 Gif-sur-Yvette Cedex, \\ France \\ 3 DSM/DAPNIA/Service d'Électronique des Détecteurs et d'Informatique, CEA/Saclay, 91191 Gif-sur-Yvette Cedex, France \\ 4 Max-Planck-Institut für Astrophysik, Karl-Schwarzschild-Str. 1, Postfach 1317, 85741 Garching, Germany
}

Received 18 November 2005 / Accepted 10 April 2006

\begin{abstract}
Context. The detection and identification of oscillation modes (in terms of their $\ell, m$, and successive $n$ ) is a great challenge for present and future asteroseismic space missions. "Peak tagging" is an important step in the analysis of these data to provide estimations of stellar oscillation mode parameters, i.e., frequencies, rotation rates, and further studies on the stellar structure.

Aims. Our goal is to increase the signal-to-noise ratio of the asteroseismic spectra computed from the time series that are representative of MOST and CoRoT observations (30- and 150-day observations).

Methods. We apply the curvelet transform - a recent image processing technique that looks for curved patterns - to echelle diagrams built using asteroseismic power spectra. In the resulting diagram, the eigenfrequencies appear as smooth continuous ridges. To test the method, we use Monte-Carlo simulations of several sun-like stars with different combinations of rotation rates, rotation-axis inclination, and signal-to-noise ratios.

Results. The filtered diagrams enhance the contrast between the ridges of the modes and the background, allowing a better tagging of the modes and a better extraction of some stellar parameters. Monte-Carlo simulations have also shown that the region where modes can be detected is enlarged at lower and higher frequencies compared to the raw spectra. In addition, the extraction of the mean rotational splitting from modes at low frequency can be done more easily using the filtered spectra rather than the raw spectra.
\end{abstract}

Key words. stars: oscillations - methods: data analysis - techniques: image processing

\section{Introduction}

Helioseismology - the study of solar oscillations - is a powerful probe of the structure and dynamics of the Sun that has provided great improvements in our understanding of stellar evolution and structure (Turck-Chièze et al. 1993; Christensen-Dalsgaard 2002, and references therein). Those successes push the community to apply seismic techniques to other stars, opening the doors to asteroseismology, the study of stellar oscillations. These oscillations have already been observed from the ground and space. The ground-based observations are limited by the daynight cycle, which introduces aliases in the observations, but they allow us to use Doppler velocity measurements. They have provided data with sufficient quality to detect solar-like oscillations (see Bouchy \& Carrier 2003; Bedding \& Kjeldsen 2003, and references therein). To reduce the aliases, multi-site campaigns have been carried out, but they are too short to have good frequency resolutions. Space photometry missions and groundbased velocity networks must be used to provide observations of stellar oscillations without these limitations. With the current

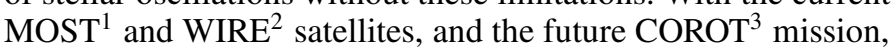
asteroseismology is blooming. However, in the case of solar-like oscillations, we still have to deal with very small signal-to-noise

\footnotetext{
1 Microvariability and Oscillations of Stars (Matthews 1998).

2 Wide-field Infrared Explorer (Buzasi et al. 2000).

${ }^{3}$ Convection Rotation and planetary Transits (Baglin et al. 2001).
}

ratio (hereafter $S / N$ ) observations as a consequence of the weakness of the luminosity variations. Moreover, stars cannot yet be spatially resolved. Instead, only global oscillation modes can be observed. In addition, we cannot have access to the rotation rates and the rotation-axis inclination separately. Without knowing these two key stellar properties, the tagging of the modes in terms of their properties $(\ell, m)$ and successive $n$ may be extremely difficult. In fact, the main problem to overcome will not be fitting the peaks ("peak-bagging"), but providing a good description of the model to be fitted, after having put the correct labels on the modes ("peak tagging"). Using the echelle diagram, where the modes follow ridges depending on the stellar properties, is one method that has been proposed to provide such description. To improve the $S / N$ ratio, Bedding et al. (2004) suggested filtering this diagram by a vertical smoothing. However the smoothing works well only when the ridges are quasi-vertical, which requires a very good a priori knowledge of the large difference and is restricted to the asymptotic part of the spectrum. We propose, here, to follow a similar approach, but using new mathematical denoising techniques better suited to the study of curved ridges.

At the end of the last decade, the application of mathematical transforms based on wavelets to analyze astronomical images was widely developed. The first wavelet algorithms were well-adapted for treating images with isotropic elements. However, this description presented a limitation in the context 
of astrophysics, where objects such as filaments or spiral structures exhibit a highly anisotropic character (in shape and scale). New transforms, the ridgelet (Candès 1998) and curvelet transforms (Candès \& Donoho 1999; Starck et al. 2002), were then developed to deal efficiently with such objects. Astrophysical applications (image denoising) of this technique have been presented in Starck et al. $(2003,2004)$ to analyze images of gravitational arcs, the rings of Saturn, or the CMB (Cosmic Microwave Background) map.

In this paper, we suggest using the curvelet transform to analyze asteroseismic observations (more precisely the stellar echelle diagrams), to improve the "peak tagging" of the oscillation modes and even the resultant "peak bagging". To illustrate the application of this denoising technique in the asteroseismic case, we performed Monte-Carlo simulations of ideal asteroseismic data contaminated by different levels of stochastic noise. We begin Sect. 2 with a short summary of the properties of stellar oscillation modes in the solar-like case and the construction of the echelle diagram. In Sect. 3, we introduce multiscale transforms, in particular the ridgelet and the curvelet transforms. In Sect. 4, the simulated data of a star with an oscillation spectrum similar to the Sun, but with different rotation axis inclinations and rotation rates, are presented. In Sect. 5, we discuss the results obtained in the simulations.

\section{Properties of solar-like oscillations}

Only low-degree stellar oscillation modes can be detected and observed with the present generation of instruments. The asymptotic theory of oscillation modes $(n \gg \ell)$ is then adequate and can be used to study them. First order (Tassoul 1980) and second order developments (Vorontsov 1991; Lopes \& Turck-Chièze 1994; Roxburgh \& Vorontsov 2000a,b) have been made to describe solar and stellar oscillations. In the case of solar-like stars, where $p$-modes predominate, the frequencies can be developed as:

$v_{n, \ell} \approx \Delta v_{0}\left(n+\frac{\ell}{2}+\frac{1}{4}+\alpha(v)\right)+\frac{\Delta v_{0}}{4 \pi^{2} v_{n, \ell}}\left((\ell+1 / 2)^{2} A+\psi\right)$,

in this expression, $\ell$ and $n$ are, respectively, the degree and the radial order of the modes and

$$
\begin{aligned}
\tau_{\mathrm{c}} & =\int_{r_{\text {in }}}^{r_{\text {out }}} \frac{\mathrm{d} r}{c_{\mathrm{s}}} \\
\Delta v_{0} & =\frac{1}{2 \tau_{\mathrm{c}}} \\
A & =\frac{1}{4 \pi^{2} v_{n, \ell}}\left(\frac{c_{\mathrm{s}}\left(R_{\star}\right)}{R_{\star}}-\int_{r_{\text {in }}}^{r_{\text {out }}} \frac{\mathrm{d} c_{\mathrm{s}}}{\mathrm{d} r} \frac{\mathrm{d} r}{r}\right),
\end{aligned}
$$

where $c_{\mathrm{s}}$ is the internal stellar sound speed, $\alpha$ is a phaseshift term, and $\psi$ is a function that allows us to take the gravitational potential in the central region into account (Lopes \& Turck-Chièze 1994). From the asymptotic approach, we can extract general properties of modes and better understand the physics hidden in the frequencies' behavior. The large frequency spacing, defined as $\Delta v_{n, \ell}=v_{n+1, \ell}-v_{n, \ell}$, tends asymptotically to $\Delta v_{0}$, related to the mass and radius of the star; the small frequency spacing, $\delta_{\ell, \ell+2} v=v_{n, \ell}-v_{n-1, \ell+2}$, can be approximated to first order by $(4 \ell+6) \Delta v_{0} /\left(4 \pi^{2} v_{n, \ell}\right) \int_{0}^{R_{\star}} \frac{\mathrm{d} c_{\mathrm{s}}}{\mathrm{d} r} \frac{\mathrm{d} r}{r}$. This variable is related to the derivative of the sound speed and enhances the effect coming from the central regions, providing constraints on the age of the star. Finally, the second difference is defined as $\delta_{2} v=v_{n+1, \ell}-2 v_{n, \ell}+v_{n-1, \ell}$. Its variations provide information
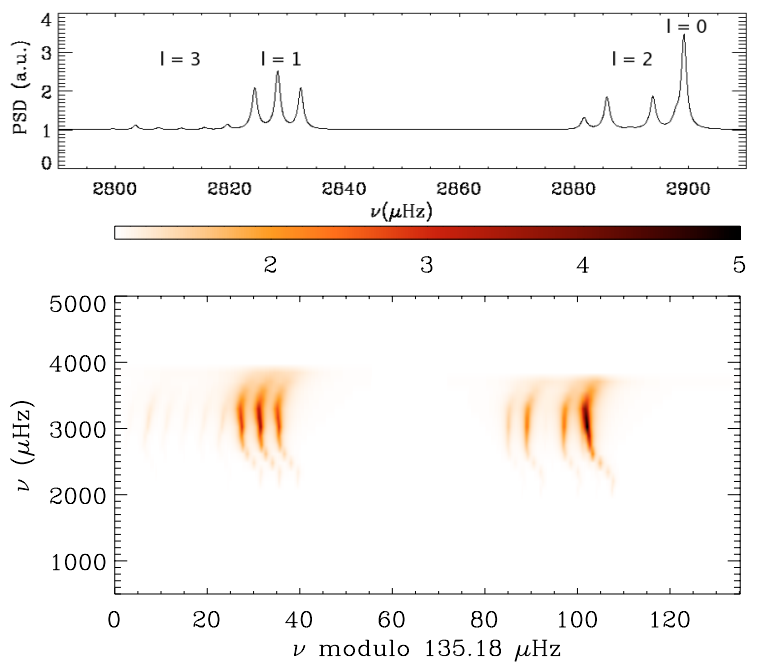

Fig. 1. Portion of the theoretical spectrum (top) and echelle diagram (bottom) for a sun spinning ten times faster than the Sun and seen from an angle of $50^{\circ}$. This is the ideal power spectrum used in the simulations described in Sect. 5.

about the extent of the convective zone (Monteiro et al. 2000; Ballot et al. 2004b) or the helium abundance in the stellar envelope (Basu et al. 2004).

Under the rotation effects, the azimuthal order $m(-\ell \leqslant$ $m \leqslant \ell)$ is needed to characterize the oscillation spectrum. If the angular velocity $\Omega$ is uniform (Ledoux 1951), the mode frequencies are asymptotically approximated by:

$v_{n, \ell, m} \approx v_{n, \ell}+m \Omega / 2 \pi=v_{n, \ell}+m \delta v$,

where $\delta v$ is the rotational splitting. Equation (2) shows that modes are $(2 \ell+1)$-times degenerated in the azimuthal order: a single peak in the spectrum becomes a multiplet. Its corresponding structure depends on the rotation rate, the inclination axis of the star, and its stochastic excitation. The solar-like mode lifetimes (a few days) are expected to be much shorter than the length of the future space observations (a few months). In consequence, the relative amplitude ratios inside a multiplet will only depend, on average, on the inclination angle and the spacing between these different $m$-components (Gizon \& Solanki 2003). Thus, if the different $m$-components of a multiplet can be identified and tagged with the correct $(\ell, m)$, they can provide a good estimation of both the rotation-axis inclination $i$ and the rotational splitting $\delta v$, allowing for a better mode parameter extraction through the fitting of the spectra. The effect of the stochastic excitation on an isolated mode could be minimized by computing the average of these parameters on several modes (see, for example, the $n$-collapsogramme; Ballot et al. 2004a).

Equation (1) shows that the even $(\ell=0,2)$ and odd $(\ell=1,3)$ modes have almost the same frequency, only separated by the small spacing $\delta_{\ell, \ell+2} v$. In addition, they are separated regularly in frequency by the large spacing $\Delta v_{n, \ell}$. This property allows us to build the so-called echelle diagram (Grec et al. 1983), which is currently used to identify modes for solar-like oscillations. It is a $2 \mathrm{D}$ representation of the $1 \mathrm{D}$ power spectrum that has been folded onto itself in units of the large spacing. In such a representation the modes appear as almost locally vertical ridges (see Fig. 1). The echelle diagram is a powerful tool for "peak tagging", since assigning the correct $(\ell, m)$ values to the peaks is easier when the multiplet structure is well-identified in this diagram. The successive $n$ values are obtained from each individual horizontal line. 


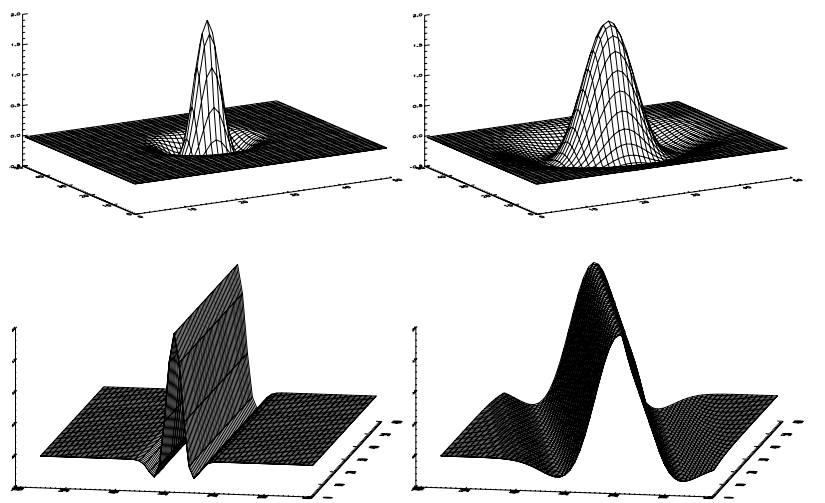

Fig. 2. Examples of 2D wavelets (top panels) and ridgelets (bottom panels). The top right wavelet has a greater scale parameter than that on the left. The bottom right ridgelet has different orientation and width than the left one.

\section{Multiscale transforms}

\subsection{The wavelet transform}

The wavelet transform provides a framework for decomposing images into their elementary constituents across scales, by reducing the number of significant coefficients necessary to represent an image. The continuous wavelet transform of a $2 \mathrm{D}$ signal is defined as:

$W\left(a, b_{i}, b_{j}\right)=\frac{1}{\sqrt{a}} \iint f(x, y) \psi^{*}\left(\frac{x-b_{i}}{a}, \frac{y-b_{j}}{a}\right) \mathrm{d} x \mathrm{~d} y$,

where $W(a, b)$ are the wavelet coefficients of the function $f(x)$, $\psi(x)^{*}$ is the conjugate of the analyzing wavelet, $a>0$ is the scale parameter, and $b$ is the position parameter. The continuous wavelet transform is the sum over all the positions of the signal $f(x, y)$, multiplied by the scaled and shifted versions of the wavelet $\psi\left(\left(x-b_{i}\right) / a,\left(y-b_{j}\right) / a\right)$ (cf. Fig. 2, top panels). This process produces wavelet coefficients that are a function of scale and position.

However, the classical wavelet transform only addresses a portion of the whole range of interesting phenomena: isotropic features at all scales and locations. One of the drawbacks of the two-dimensional wavelet transform is that it does not achieve an efficient analysis of images that present high anisotropy. For instance, the wavelet transform does not efficiently approximate 2D edges, since a large number of large wavelet coefficients, scale after scale, are required, making its analysis difficult. To solve this problem, two new mathematical transforms, namely the ridgelet transform and the curvelet transform, were introduced.

\subsection{The ridgelet transform}

The ridgelet transform was developed to process images including ridges elements (Candès 1998). It provides a representation of perfectly straight edges. A given function $f\left(x_{1}, x_{2}\right)$ can be represented as the superposition of elements of the form $a^{-1 / 2} \psi\left(\left(x_{1} \cos \theta+x_{2} \sin \theta-b\right) / a\right)$, where $\psi$ is a wavelet, $a>0$ a scale parameter, $b$ a location parameter, and $\theta$ an orientation parameter. The ridgelet is constant along the lines where $x_{1} \cos \theta+x_{2} \sin \theta=$ const., and transverse to these ridges it is a wavelet. Thus, contrary to a unique wavelet transform, the ridgelet has two supplementary characteristics: a length, equal to that of the image, and an orientation, allowing the analysis of

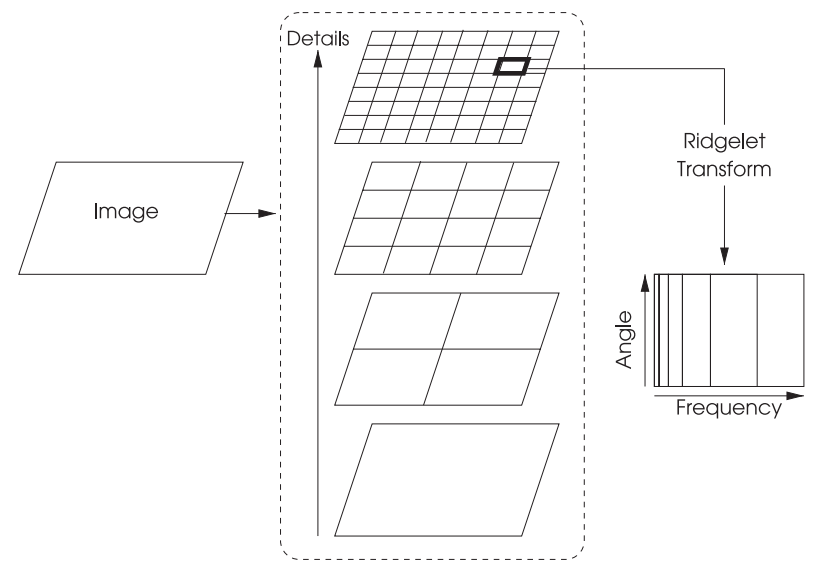

Fig. 3. Sketch illustrating the curvelet transform applied to an image. The image is decomposed into subbands followed by a spatial partitioning of each subband. The ridgelet transform is applied to each block. The finest details correspond to the highest frequencies.

an image in every direction and thus exhibiting the edge structure. Figure 2 (bottom panels) shows two examples of ridgelets. The problem is that in nature, edges are typically curved rather than straight, so ridgelets alone cannot yield accurate representations.

\subsection{The curvelet transform}

\subsubsection{Description}

Ridgelets can be adapted to represent objects with curved edges, using an appropriate multiscale localization: at a sufficiently fine scale, a curved edge can be considered to be almost straight. Candès \& Donoho (1999) developed the curvelet transform using ridgelets in this localized manner. Figure 3 shows the different steps of the curvelet analysis of an image:

1. Image decomposition: using a $2 \mathrm{D}$ isotropic wavelet transform, the image is decomposed to obtain a set of wavelet bands. Each band corresponds to a different scale.

2. Smooth partitioning: each subband is partitioned into squares (blocks), whose size is appropriate to each scale. The finer the scale is, the smaller the blocks are.

3. Ridgelet analysis: it is applied to each square.

The implementation of the curvelet transform offers an exact reconstruction and a low computational complexity. Like ridgelets, curvelets occur at all scales, locations, and orientations. Moreover, contrary to ridgelets, which have a given length (the image size) and a variable width, the curvelets also have a variable length (the block size) and, consequently, a variable anisotropy. The finer the scale is, the more sensitive to the curvature the analysis is. As a consequence, curved singularities can be well-approximated with very few coefficients.

\subsubsection{Denoising images: filtering curvelet coefficients}

To remove noise, a simple thresholding of the curvelet coefficients has been applied to select only significant coefficients. One possible thresholding of a noisy image consists of setting all non-significant curvelet coefficients $\tilde{c}_{i, j, l}, i, j$, and $l$ respectively, the indexes of the line, row, and scale to 0 . This is called hard-thresholding:

$\tilde{c}_{i, j, l}= \begin{cases}1 & \text { if } c_{i, j, l} \text { is significant } \\ 0 & \text { if } c_{i, j, l} \text { is not significant. }\end{cases}$ 
Commonly, $c_{i, j, l}$ is significant if the probability that the curvelet coefficient is due to noise is small, i.e., if the curvelet coefficient is greater than a given threshold. A basic problem remains: the choice of the threshold. Usually, this threshold is equal to $k \sigma_{j}$, where $\sigma_{j}$ is the noise standard deviation at the scale $j$ and $k$ is a constant, equal to 5 in our filterings.

Simple thresholding of the curvelet coefficients is very competitive (Starck et al. 2002) with "state of the art" techniques based on wavelets, including thresholding of decimated or undecimated wavelet transforms.

\section{Simulation of data}

To characterize the curvelet denoising technique applied to the asteroseismic data, we have simulated typical solar-like observations while varying different parameters: $S / N$ ratios, observational lengths, rotation-axis inclinations, rotation rates, etc. With this approach, we know the input parameters in advance, and we can evaluate the quality of the results given by the curvelet analysis and its limits.

In the simulations shown in this paper, we use the oscillation spectrum of a star similar to the Sun, but seen under different conditions. We consider different rotation-axis inclinations $\left(i=50^{\circ}\right.$ and $\left.90^{\circ}\right)$ and rotation rates $\left(\Omega=\Omega_{\odot}, 5 \Omega_{\odot}\right.$, and $10 \Omega_{\odot}$ ). An ideal power spectrum was constructed first. Only the modes $\ell \leq 3, n=12-25$ were simulated. The mode parameters - frequencies $(v)$, amplitudes $(A)$, and widths $(\Gamma)-$ were obtained from the analysis of GOLF (Global Oscillations at Low Frequency) data (García et al. 2004). The amplitudes were corrected to take into account the difference between intensity and velocity observations. Modes were simulated with symmetrical Lorentzian profiles as the asymmetry is expected to be at the level of the noise. Following the method described in Fierry Fraillon et al. (1998), a multiplicative noise, a $\chi^{2}$ with 2 d.o.f. statistics, has been introduced to reproduce the stochastic excitation of such modes (see also Anderson et al. 1990). The $S / N$ ratio of the "resultant" raw power spectrum was defined as the maximum of the bell-shaped $p$-mode power (i.e., the highest simulated $p$-mode) divided by the noise dispersion. The simulated background is flat, assuming that it has been previously fitted and removed, as it is usually done for the Sun (Harvey 1985).

Several Monte-Carlo simulations have been performed for each ideal spectrum. Realistic $S / N$, with values ranging from 5 to 15 , have been used to cover a wide range of situations that is compatible with what is expected (see Baglin et al. 2001). In each realization of the Monte-Carlo simulation, the same level of noise has been randomly added to the corresponding ideal spectra. Therefore, all the realizations, in a given Monte-Carlo simulation, have the same $S / N$ ratio. The simulated spectra have been computed for two resolutions, $\approx 0.38$ and $\approx 0.077 \mu \mathrm{Hz}$, corresponding respectively to 30 -day and 150 -day observations. The first are representative of the MOST observations and the short CoRoT runs, while the latter are of the same length as the long CoRoT runs.

Simulations of other stars, including some potential main CoRoT targets, with different masses, ages, and, in consequence, internal structures, have been made. The results have already been presented and discussed during the CoROT workshops \#8 and \#9 obtaining the same qualitative results. For the sake of clarity, they are not shown here.

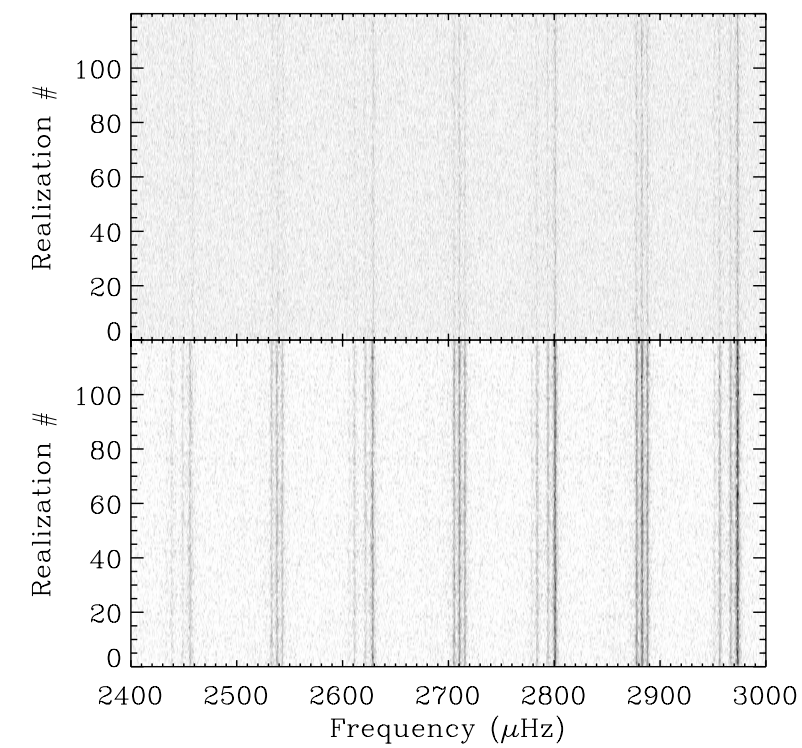

Fig. 4. Effect of the curvelet denoising on the mode visibility for $S / N=5$. Each picture shows 120 realizations out of the 500 done in our Monte-Carlo simulation. Each horizontal line corresponds to a single realization. The top panel is the raw spectra, and the bottom is the curvelet filtered one.

\section{Discussion}

Once the spectra have been computed, the echelle diagrams can be built with a fixed folding frequency. This frequency corresponds to the mean large frequency spacing $\Delta v_{0}$, identified either by the computation of the FFT, the autocorrelation of the spectra, or any other technique (see for example Régulo \& Roca Cortés 2002). The denoising based on the curvelet transform is then applied to these echelle diagrams. It is important to note that artifacts may appear in the filtered spectra at frequencies $v^{*}=v_{0}+k \Delta v_{0}$, with an integer $k$, when random small structures appear in the echelle diagrams. However, their appearance and position strongly depend on the folding frequency and are very sensitive to its value. Therefore, they can be easily identified. The artifacts can be reduced (in contrast to the regions containing the signal) by building echelle diagrams with slightly different folding frequencies and averaging the resultant filtered spectra.

To present the results of data analysis using the curvelet denoising method, we have selected the case of a sun-like star seen with an inclination angle $i=50^{\circ}$ and with a rotation $\Omega=10 \Omega_{\odot}$. A portion of the ideal spectra constructed for this star can be seen in Fig. 1 (top panel). Monte-Carlo simulations were then performed, giving rise to different sets (each one with 500 realizations) of raw spectra with different $S / N$ ratios. The echelle diagrams were constructed using a folding frequency of $135.18 \mu \mathrm{Hz}$, obtained by computing the FFT of the raw spectrum.

\subsection{Peak tagging}

In the cases with a high $S / N$ (typically 15), the mode structure is clearly visible in each raw spectrum, as well as on the echelle diagram. The different ridges can be easily identified and tagged. Although the filtering gives enhanced denoised diagrams and unfolded spectra, it does not contribute significantly to the mode identification.

In the lower $S / N$ cases, however, the situation is different. Figure 4 shows some of the results of the Monte-Carlo 

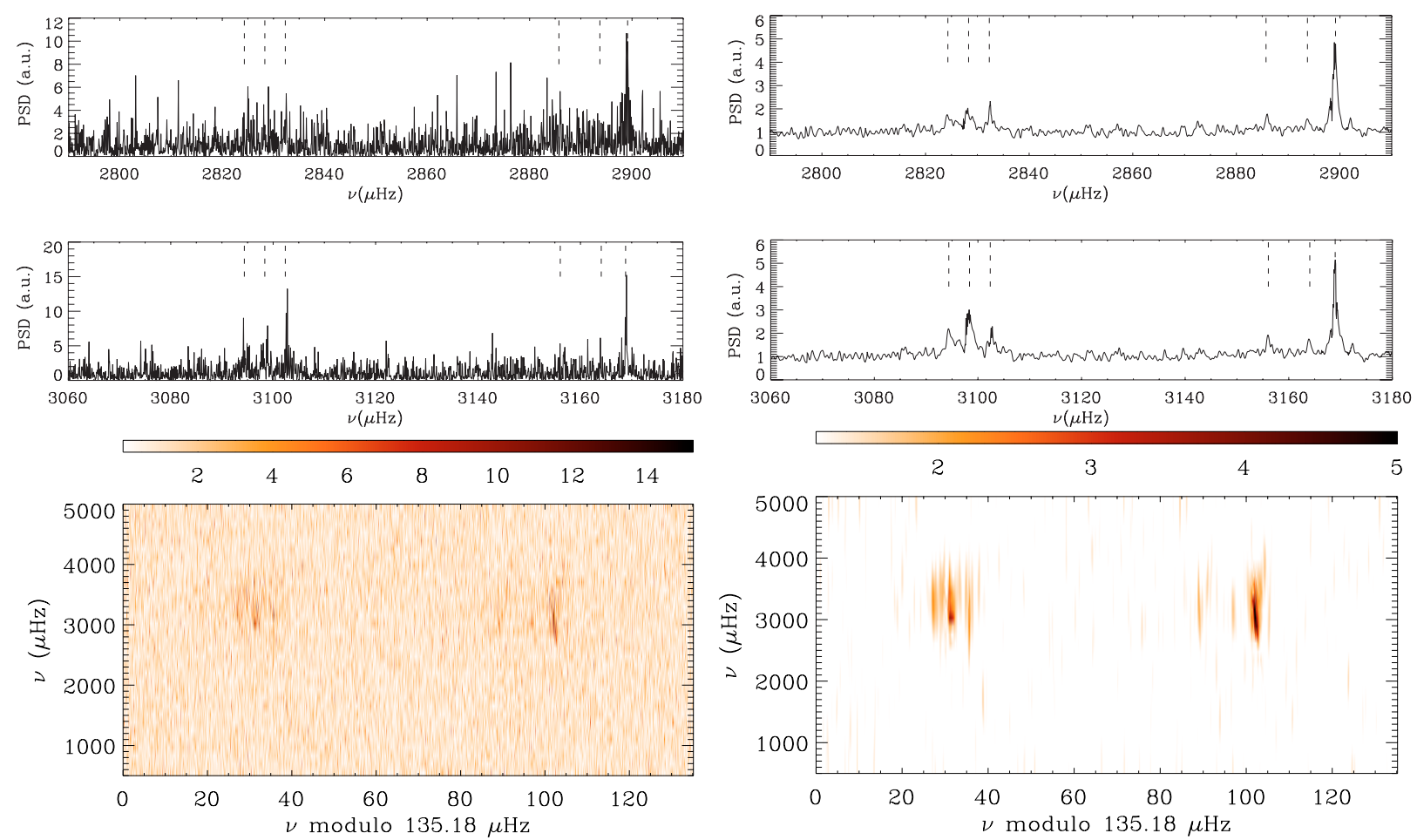

Fig. 5. Raw (left) and filtered (right) power spectra (top and middle panels) and echelle diagrams (bottom panels) for a $S / N=5$ realization. The short dashed lines in the power spectra represent the position of the theoretical frequencies. From left to right, the three first equidistant lines indicate the components $m=-1,0$, and 1 of $\ell=1$ modes, the next two indicate the strongest components of $\ell=2(m=-1$ and 1$)$, and the the last indicates $\ell=0$. In this case, only two components of the $\ell=1$ and the $\ell=0$ modes are slightly visible in the raw diagram. On the curvelet filtered one, the three $\ell=1$ components appear as well as the $\ell=0$ and the components $m= \pm 1$ of the $\ell=2$ modes.

simulation for $S / N=5$. The upper panel corresponds to 120 realizations among the 500 computed for the raw spectra in the frequency range $2450-2920 \mu \mathrm{Hz}$. Each horizontal line corresponds to a single realization. Some patterns can hardly be seen. The lower panel represents the same spectra after applying the curvelet filtering. A series of vertical ridges clearly appears. From left to right on the panels, they can be identified as the $(\ell=2 ; m= \pm 1)$, the $\ell=0$ (blended with the $\ell=2 ; m=+2$ ), and the $(\ell=1 ; m=-1,0,+1)$. The improvement of the contrast is important in all the realizations and allows us to distinguish the different components of a mode, making the identification and the tagging easier.

The identification is harder when looking at each individual spectrum and requires the use of the echelle diagram. Figure 5 shows an example of raw (left) and filtered (right) 150-day observation power spectra (top and middle panels), and the corresponding echelle diagrams (bottom panels) for a $S / N=5$ realization. Input frequencies are indicated by the short dashed lines above the spectra. The mode peaks can hardly be distinguished in the raw spectrum and can easily be confused with noise. For the range $2780-2920 \mu \mathrm{Hz}$, only a strong peak at $2900 \mu \mathrm{Hz}$ can be considered not to be noise. In the region $3060-3180 \mu \mathrm{Hz}$, the peaks are visible, and we can attempt to identify the $\ell=1$ and $\ell=0$ modes, although they are still unclear. On the contrary, on the corresponding parts of the filtered spectrum, the structures of the $\ell=1$ mode with three components, the $\ell=0$ mode, and even the strongest components of the $\ell=2$ mode are visible. The raw echelle diagram gives no extra information because of the very weak ridges and low contrast with the background. The weakest components can hardly be detected, and no tagging can be done. The curvelet filtering provides a contrast enhancement of the ridges on the echelle diagram. Thus, three almost equidistant strong ridges appear on the left of the diagram and one strong ridge with two weaker ones on the right. The corresponding patterns can be seen on the filtered spectrum corresponding well to the theoretical frequencies. Since the modes $\ell=3$ are not visible, and in agreement with the amplitude of the strongest peak on the left, we can suggest that the three strongest peaks correspond to a $\ell=1$ multiplet and the other ones to the $\ell=2$ and $\ell=0$ modes.

Consequently, when the tagging is done, it is also easier to have a first estimation of both the mean rotational splitting and the rotation-axis inclination, since the visibility of the multiplet is increased. From the spacing between the components of the mode $\ell=1$, a first estimation of the mean rotational splitting of the star, as well as an estimation of the inclination angle, according to their relative amplitude ratios, can be done. We have selected the extraction of one parameter: the mean rotational splitting of the $\ell=1$ mode at low frequency $(2540-2550 \mu \mathrm{Hz})$, to quantify the improvement of the curvelet filtering. This region is particularly interesting because the line width is still small, and the modes, when they are visible, can be easily identified. Thus, in a sample of 100 realizations of the Monte-Carlo simulation, we obtained a better estimation of this parameter in the filtered spectra in 90 of them. In fact, it was very exceptional to obtain a good result in the raw spectra. With the filtered spectra, a mean rotational splitting of $\langle\delta v\rangle=4.05 \pm 0.30 \mu \mathrm{Hz}$ was found, which is very close to the actual splitting included in the ideal spectra $\langle\delta v\rangle=4.0 \mu \mathrm{Hz}$. In addition, specific methods can be applied to improve the extraction of these parameters by using different strategies of spectra fitting, such as the ones developed by Gizon \& Solanki (2003) or Ballot et al. (2006). In the case of the 30-day observation, the curvelet filtered echelle diagram is still very noisy, and it does not help in recognizing the ridges. 

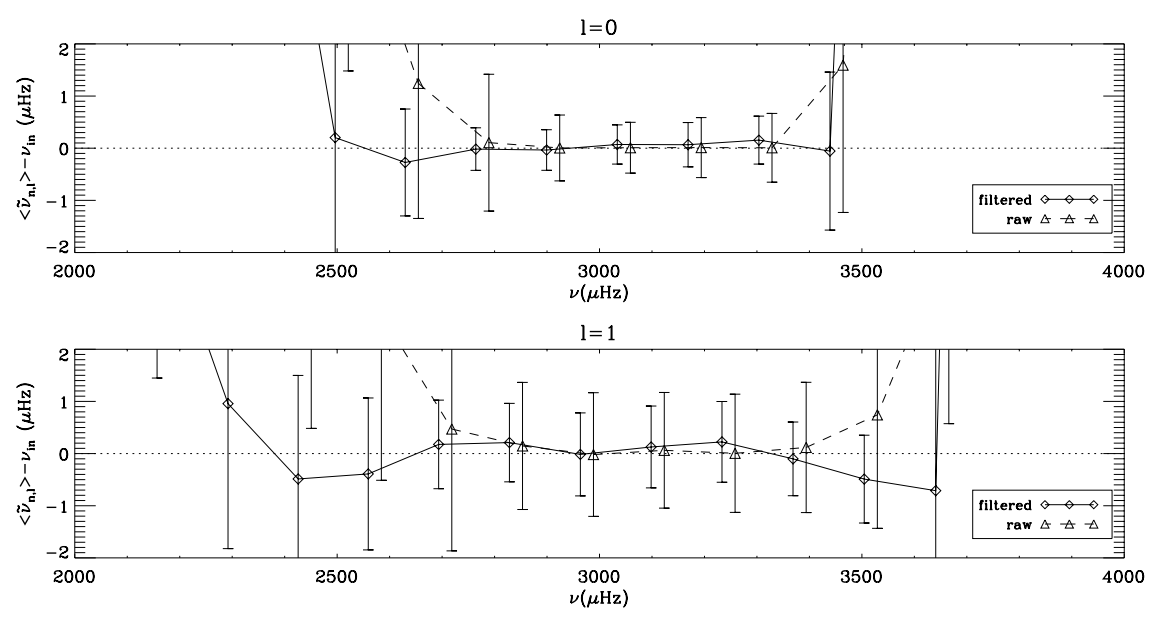

$1=2$

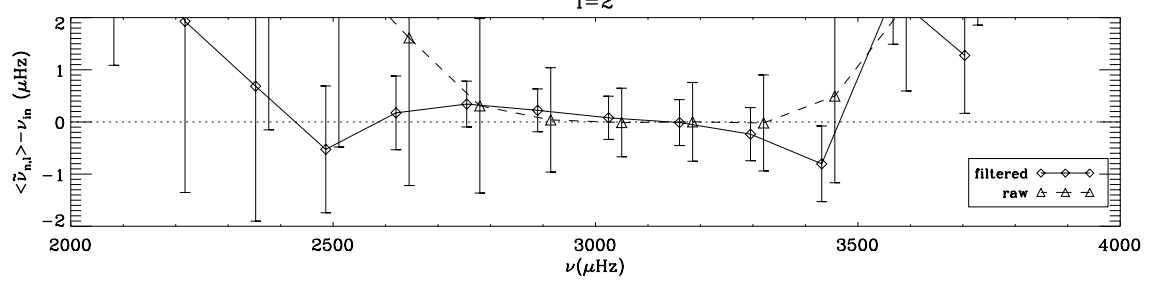

Fig. 6. Differences between the mean fitted frequencies $\left\langle\tilde{v}_{n, \ell}\right\rangle$ and the input frequencies $v_{\text {in }}$, for $\ell=0$, 1, and 2 , for the raw (dashed line with triangles) and filtered (full line with diamonds) spectra $\left(S / N=5,150\right.$-day observation). The error bars correspond to the dispersion $\sigma^{*}\left(\tilde{v}_{n, \ell}\right)$ of the frequency distribution. For clarity, the values for the raw case are shifted by $20 \mu \mathrm{Hz}$ towards the right.

However, the corresponding denoised power spectrum is much better, despite the lower resolution ( 5 times less than in the long runs), even for small $S / N$ ratios $(\sim 5)$. The modes $\ell=0,2$ and $\ell=1$ can be distinguished, at the maximum power, while it is not easy to do so in the raw spectra. Therefore, we consider that a 30 -day run is the minimum length needed to have reliable results with the curvelet denoising technique.

García et al. (2006) analyzed the first available MOST public Procyon A data (32-day observation) using the curvelet technique. Previous analysis by Matthews et al. (2004) did not reveal the presence of any $p$-mode structure in this star. Therefore, due to its tiny $S / N$ ratio, the results of the curvelet denoising should be taken with care. Nevertheless, an excess of power seems to appear in the region where it is expected, and taking the 15 most prominent peaks in this region, many are in agreement, inside the error bars, with previous tagged modes using ground-based velocity observations.

\subsection{Extraction of $p$-mode parameters}

Once the mode identification and tagging are done, the extraction of the mode parameters can be performed. To illustrate how this extraction can be improved by using the denoised spectrum, we have extracted the central frequency of the modes in both the raw and the filtered spectra. To determine this parameter, modes have been fitted by Lorentzian profiles using a maximumlikelihood estimator in the classical way: adjacent pairs of even ( $\ell=0$ and $\ell=2$ ) modes are fitted together, while $\ell=1$ is fitted alone, due to the small amplitudes of $\ell=3$ modes. For each multiplet, the fitted parameters are the central frequency $\tilde{v}_{n, \ell}$, the amplitude $\tilde{A}_{n, \ell}$, the linewidth $\tilde{\Gamma}_{n, \ell}$, and the background $b$. The amplitude ratios inside the multiplets and the rotational splittings have been fixed thanks to the preliminary estimation done in the previous section (cf. Sect. 5.1). The fitting procedure provides an associated error $\sigma(\tilde{X})$, computed by Hessian-matrix inversion, for each adjusted parameter $\tilde{X}$.

The raw spectra follow a $\chi^{2}$ with 2 d.o.f. statistics, whereas the filtered spectra have a $\chi^{2}$ with a higher d.o.f. statistics (close to a Gaussian distribution, depending on the number of filtered coefficients). According to Appourchaux (2003), it is possible to fit spectra following a $\chi^{2}$ with more than 2 d.o.f. statistics with a classical procedure developed for a $\chi^{2}$ with 2 d.o.f. statistics: parameters are correctly fitted, but computed errors have to be adapted a posteriori. However in our case, due to filtering, points of filtered spectra are correlated (we have estimated that one point is correlated with $\sim 10$ neighboring points). This correlation should have to be considered, but we have neglected its effect on the fitting procedure in the present study. This assumption is validated by the Monte-Carlo simulations. Such a global filtering also induces correlations between the different lines of the echelle diagram. Thus, the errors on parameters of different modes (typically $(n, \ell)$ and $(n+1, \ell))$ can be correlated. These correlations will have to be taken into account, especially during the comparison of frequencies extracted in this way from stellar models.

From the 500 realizations of the Monte-Carlo simulation, we derived the mean value of the extracted frequencies $\left\langle\tilde{v}_{n, \ell}\right\rangle$, their mean computed errors $\left\langle\sigma\left(\tilde{v}_{n, \ell}\right)\right\rangle$, and the dispersion of the frequency distribution $\sigma^{*}\left(\tilde{v}_{n, \ell}\right)$ (the real error), for each mode and for both the raw and the filtered spectra. We have verified that $\sigma^{*}\left(\tilde{v}_{n, \ell}\right) \approx\left\langle\sigma\left(\tilde{v}_{n, \ell}\right)\right\rangle$ for fits performed on the raw spectra, and we have $\sigma^{*}\left(\tilde{v}_{n, \ell}\right)<\left\langle\sigma\left(\tilde{v}_{n, \ell}\right)\right\rangle$ for fits performed on the filtered ones. As expected, the error bars on the fitted frequencies, computed by Hessian-matrix inversion, are overestimated.

Figure 6 shows the difference between the mean fitted frequencies $\left\langle\tilde{v}_{n, \ell}\right\rangle$ and the theoretical frequencies $v_{i n}$ of the simulated star discussed in the previous section $(S / N=5)$. The error bars correspond to the dispersion $\sigma^{*}\left(\tilde{v}_{n, \ell}\right)$. For each $\ell$, the error bars of the filtered spectra are smaller than those of the raw 
spectra. In addition, the range where modes can be detected, tagged, and fitted is extended. While the difference $\left\langle\tilde{v}_{n, \ell}\right\rangle-v_{\text {in }}$ is only flat in the central region of the raw power spectrum (e.g., for $\ell=0$, in the range $n=18-22$ ), it extends at higher and lower frequencies (e.g., for $\ell=0$, the range is extended to $n=16-23$ ) in the filtered one.

\section{Conclusions}

The application of a noise reduction technique based on the curvelet transform to echelle diagrams improves the identification - "peak tagging" - of stellar acoustic modes. In observations with a $S / N$ ratio as small as 5 , we are still able to recover the mode pattern and extract reliable asteroseismic information in both small and long runs (30-day and 150-day observations, respectively). Below this $S / N$ and with shorter observations, the method efficiency is reduced drastically. The rotational splittings and the rotation-axis inclination can be better estimated using the filtered spectrum. In particular, Monte-Carlo simulations showed that a better extraction of the mean rotational splitting from modes at low frequency can be done in 90 out of 100 realizations using the filtered spectra. The uncertainty on the extracted rotational splitting of a typical sun-like star seen with an inclination angle $i=50^{\circ}$ and with a rotation $\Omega=10 \Omega_{\odot}$ is very small, $\sim 0.30 \mu \mathrm{Hz}$. These parameters can then be used to create a set of guesses or a priori values to perform individual fits of the spectra. We have also shown that the range of the frequency extraction can be extended at higher and lower frequencies using the filtered spectra. Finally, simulations of the short run observations have demonstrated that this method can also be applied to lower resolution spectra with good results.

Acknowledgements. P. Lambert thanks Dr. D. Neuman for useful discussions.

\section{References}

Anderson, E. R., Duvall, T. L., \& Jefferies, S. M. 1990, ApJ, 364, 699 Appourchaux, T. 2003, A\&A, 412, 903
Baglin, A., Auvergne, M., Catala, C., Michel, E., \& COROT Team. 2001, in SOHO 10/GONG 2000 Workshop: Helio- and Asteroseismology at the Dawn of the Millennium, ESA SP-464, 395

Ballot, J., García, R. A., Lambert, P., \& Teste, A. 2004a, in SOHO 14 Helio- and Asteroseismology: Towards a Golden Future, ESA SP-559 309

Ballot, J., Turck-Chièze, S., \& García, R. A. 2004b, A\&A, 423, 1051

Ballot, J., García, R. A., \& Lambert, P. 2006, MNRAS, 369, 1281

Basu, S., Mazumdar, A., Antia, H. M., \& Demarque, P. 2004, MNRAS, 350, 277

Bedding, T. R. \& Kjeldsen, H. 2003, Publ. Astron. Soc. Austr., 20, 203

Bedding, T. R., Kjeldsen, H., Butler, R. P., et al. 2004, ApJ, 614, 380

Bouchy, F. \& Carrier, F. 2003, Ap\&SS, 284, 21

Buzasi, D., Catanzarite, J., Laher, R., et al. 2000, ApJ, 532, L133

Candès, E. J. 1998, Ph.D. Thesis, Stanford University

Candès, E. J. \& Donoho, D. L. 1999, in Curves and Surfaces: Saint-Malo 1999, ed. A. Cohen, C. Rabut, \& L. Schumaker, (Nashville, TN: Vanderbilt University Press)

Christensen-Dalsgaard, J. 2002, Rev. Mod. Phys., 74, 1073

Fierry Fraillon, D., Gelly, B., Schmider, F. X., et al. 1998, A\&A, 333, 362

García, R. A., Jiménez-Reyes, S. J., Turck-Chièze, S., Ballot, J., \& Henney, C. J. 2004, in SOHO 14 Helio- and Asteroseismology: Towards a Golden Future, ESA SP-559 436

García, R. A., Lambert, P., Ballot, J., et al. 2006, submitted

Gizon, L., \& Solanki, S. K. 2003, ApJ, 589, 1009

Grec, G., Fossat, E., \& Pomerantz, M. A. 1983, Sol. Phys., 82, 55

Harvey, J. 1985, in Future Missions in Solar, Heliospheric and Space Plasma Physics, 199

Ledoux, P. 1951, ApJ, 114, 373

Lopes, I., \& Turck-Chièze, S. 1994, A\&A, 290, 845

Matthews, J. M. 1998, in Structure and Dynamics of the Interior of the Sun and Sun-like Stars SOHO 6/GONG 98 Workshop Abstract, June 1-4, 1998, Boston, Massachusetts, 395

Matthews, J. M., Kusching, R., Guenther, D. B., et al. 2004, Nature, 430, 51

Monteiro, M. J. P. F. G., Christensen-Dalsgaard, J., \& Thompson, M. J. 2000, MNRAS, 316, 165

Régulo, C., \& Roca Cortés, T. 2002, A\&A, 396, 745

Roxburgh, I. W., \& Vorontsov, S. V. 2000a, MNRAS, 317, 141

Roxburgh, I. W., \& Vorontsov, S. V. 2000b, MNRAS, 317, 151

Starck, J. L., Candès, E. J., \& Donoho, D. L. 2002, IEEE Trans. Image Proc., 11, 670

Starck, J. L., Aghanim, N., \& Forni, O. 2004, A\&A, 416, 9

Starck, J. L., Donoho, D. L., \& Candès, E. J. 2003, A\&A, 398, 785

Tassoul, M. 1980, ApJS, 43, 469

Turck-Chièze, S., Däppen, W., Fossat, E., et al. 1993, Phys. Rep., 230, 57

Vorontsov, S. V. 1991, SVA, 35, 400 